\title{
Análise da distribuição de vacinas contra o covid-19 no Cariri Paraibano a partir do desenvolvimento de um dashboard em power BI
}

\section{Analysis of covid-19 vaccine distribution in Cariri Paraibano from the development of a dashboard in power BI}

DOI: $10.46919 / \operatorname{arch} v 2 n 3-019$

Recebimento dos originais: 01/05/2021

Aceitação para publicação: 30/06/2021

\author{
Daniel Augusto de Moura Pereira \\ Doutor em Arquitetura e Urbanismo \\ Instituição: Universidade Federal de Campina Grande \\ Endereço: Centro de Desenvolvimento Sustentável do Semiárido - CDSA \\ Rua Luiz Grande, S/N - CEP 58540-000 \\ Sumé - Paraíba - Brasil
}

(83) 3353-1850

E-mail: danielmoura@ufcg.edu.br

Marcos dos Santos

Doutor em Engenharia de Produção

Instituição: Instituto Militar de Engenharia

Endereço: Ministério da Defesa - Comando da Marinha, Centro de Análises de Sistemas Navais -

CASNAV.

Praça Barão de Ladário $\mathrm{S} / \mathrm{N}^{\circ}$ - Ed. 23

Centro

20091000 - Rio de Janeiro, RJ - Brasil

Telefone: (21) 21977483

E-mail: marcosdossantos_doutorado_uff@yahoo.com.br

\section{David de Oliveira Costa}

Especialista Melhoria Contínua

Instituição: Universidade Católica de Recife

Endereço: R. do Príncipe, 526 - Boa Vista, Recife - PE, 50050-900

E-mail dcosta.doc@gmail.com

\section{RESUMO}

A Pandemia do Novo Corona Vírus mobilizou grandes laboratórios e empresas de todo o mundo em busca de uma vacina eficaz. No Brasil, a vacinação iniciou-se no começo de 2021 e avança, a passos lentos, com a aplicação de 57.691.259 de vacinas. Na Paraíba, o número de doses aplicadas é de 1.237.177, sendo, a maioria direcionada para a capital do Estado, João Pessoa. Neste sentido, este trabalho teve como objetivo analisar, a partir da construção de um Dashboard em Power BI, a distribuição de vacinas na Fase 1 no Cariri Paraibano. Para a realização do trabalho, foram coletados dados secundários oficiais sobre a distribuição de doses em uma plataforma do Governo do Estado. Os dados foram tabulados e foi gerada a inteligência da campanha de vacinação a partir do software Power BI. Os resultados mostram os grupos mais beneficiados com a vacinação, o índice de eficiência, o percentual de imunização global, entre outros indicadores chaves de performance para os 30 municípios do Cariri Paraibano.

Palavras-Chave: COVID-19; Vacina; Business Intelligence; Power BI, Cariri Paraibano. 


\begin{abstract}
The Pandemic New Corona Virus has mobilized major laboratories and companies around the world in search of an effective vaccine. In Brazil, vaccination began in early 2021 and is advancing, slowly, with the application of 57,691,259 vaccines. In Paraíba, the number of doses applied is 1,237,177, most of them directed to the state capital, João Pessoa. In this sense, this work aimed to analyze, from the construction of a Dashboard in Power BI, the distribution of vaccines in Phase 1 in Cariri Paraibano. To carry out the work, official secondary data on the distribution of doses were collected in a platform of the State Government. The data were tabulated and the intelligence of the vaccination campaign was generated using the Power BI software. The results show the groups that benefited the most from vaccination, the efficiency index, the percentage of global immunization, among other key performance indicators for the 30 municipalities of Cariri Paraibano.
\end{abstract}

Keywords: COVID-19; Vaccine; Business Intelligence; Power BI, Cariri Paraibano.

\title{
1 INTRODUÇÃO
}

De acordo com Cordeiro et al., (2020), no final de 2019, o mundo presenciou o surgimento de uma das mais alarmantes pandemias registradas na Idade Contemporânea, a Covid-19. O novo coronavírus (que no início foi temporariamente nomeado 2019-nCoV e, em seguida foi batizado de SARS-CoV-2) é responsável por causar a doença COVID-19 e já matou, no Brasil, até a data de 24 de maio de 2021, quase meio milhão de pessoas.

Neste sentido, os governos (federal, estadual e municipal) têm se mobilizado para criar medidas que auxiliem o combate ao novo COVID-19. Medidas estas que vão desde simples procedimentos de educação e/ou conscientização, como a utilização das máscaras faciais e álcool gel, como a vacinação em massa, para fins de imunização por rebanho. Entrementes, o processo tem avançado de maneira lenta. Vale salientar que segundo Gadelha (2008) e Temporão e Gadelha (2019), a acesso à vacinação é condicionado por aspectos econômicos, uma vez que a sua indústria integra o subsistema de base química e biotecnológica do Complexo Econômico-Industrial da Saúde (CEIS).

Neste sentido, dados do Ministério da Saúde apontam que, do começo da pandemia, até 23 de maio de 2021, foram distribuídas 90.664.475 doses em todo Brasil e foram aplicadas 57.691.259 de vacinas. A Figura 1 mostra o painel de monitoramento do COVID-19 do Governo Federal e seus principais números. 
Figura 1 - Painel COVID-19 Governo Federal

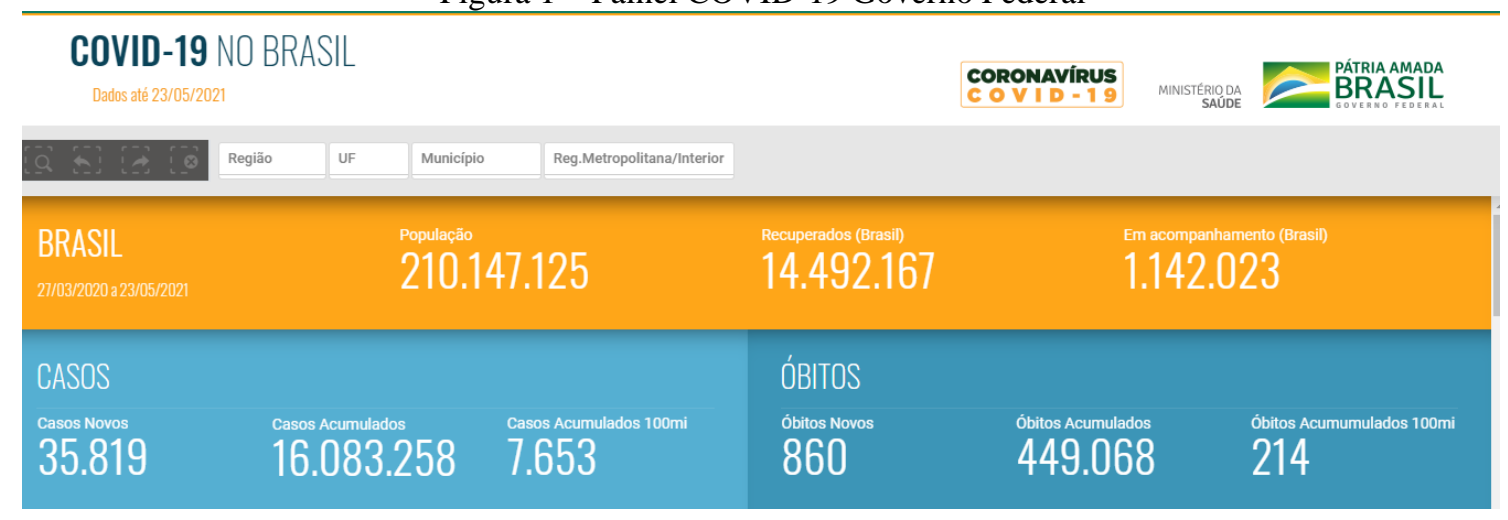

Fonte: Adaptado de https://qsprod.saude.gov.br/extensions/covid-19_html/covid-19_html.html

Na Paraíba, a Fase 1 de vacinação iniciou-se no começo do ano de 2021, assim que os primeiros imunizantes foram enviados. Verificando a mesma plataforma do Governo do Federal, a Figura 2 mostra os dados do COVID-19 para o Estado da Paraíba.

Figura 2 - Painel COVID-19 Governo Federal para a Paraíba

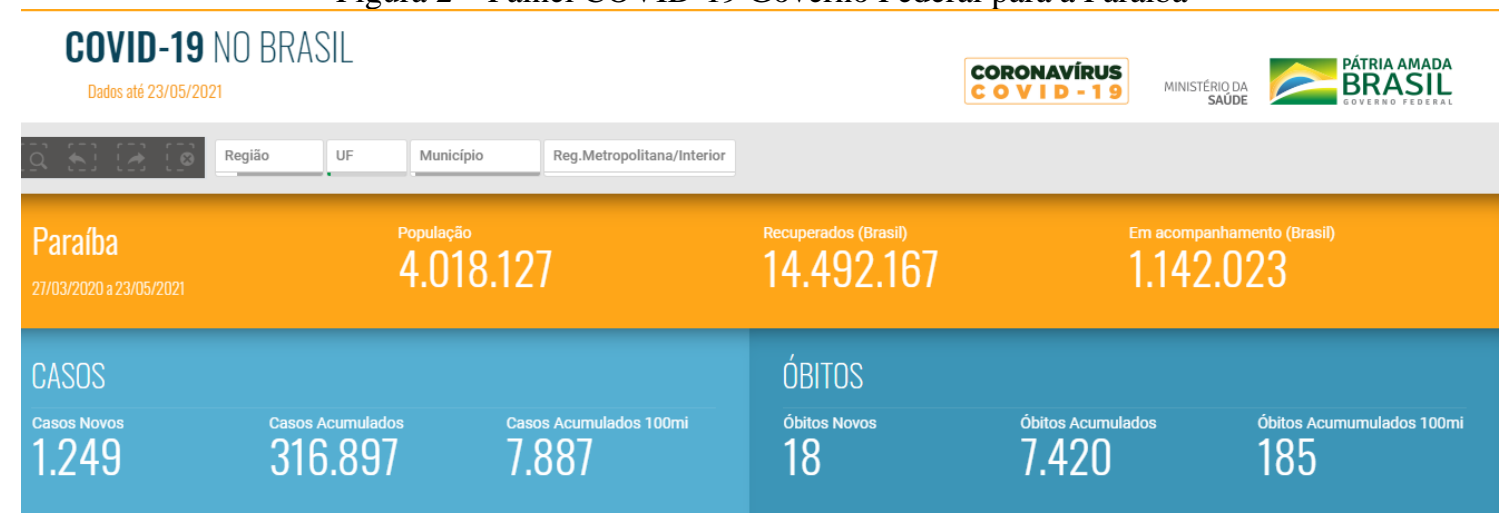

Fonte: Adaptado de https://qsprod.saude.gov.br/extensions/covid-19_html/covid-19_html.html

Observando os dados de maneira detalhada, conforme mostra a Figura 3, nota-se que a região metropolitana (Capital + 3 cidades) tem 117.978 casos e que o interior do Estado tem 198.919 casos.

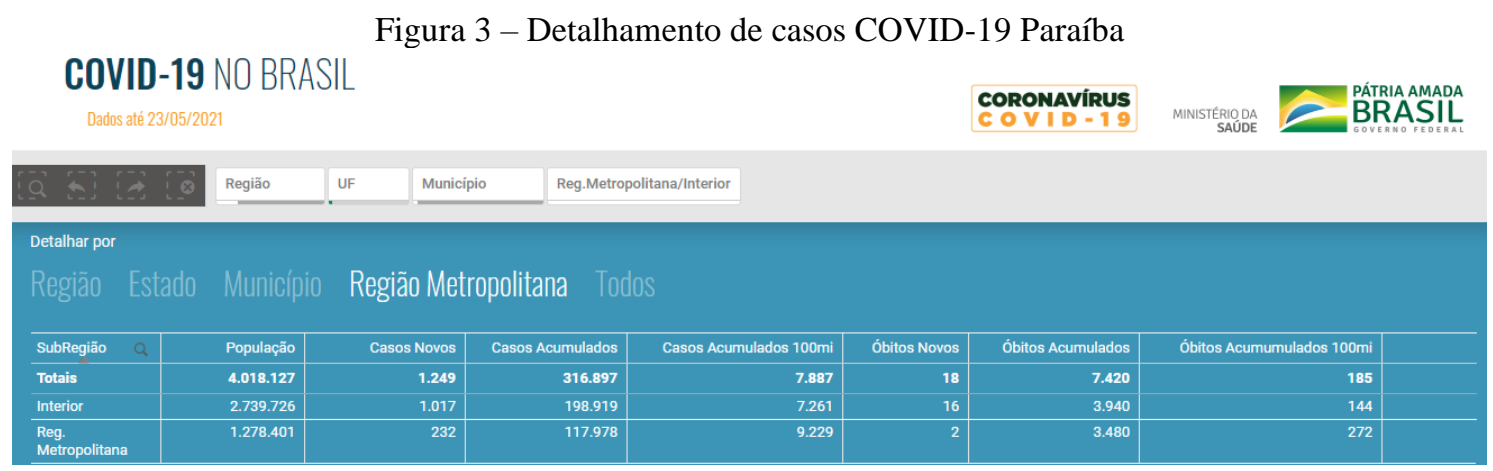

Fonte: Adaptado de https://qsprod.saude.gov.br/extensions/covid-19_html/covid-19_html.html 
O que se percebe, analisando dados fornecidos pelas autoridades locais sobre a vacinação, é que a capital do Estado, João Pessoa, tem recebido uma maior atenção do que os demais municípios do Estado. Neste sentido, este trabalho tem como objetivo analisar, a partir da construção de um Dashboard em Power BI, a distribuição de vacinas na Fase 1 no Cariri Paraibano.

\section{BUSINESS INTELLIGENCE}

Para Laudon (2014, p. 367), Business Intelligence (BI) é um termo usado por fornecedores de hardware e software e consultores de tecnologia da informação para descrever a infraestrutura para armazenamento, integração, elaboração de relatórios e análise de dados que vêm do ambiente empresarial.

Todesco et al.; (2007) apresentaram o conceito de BI como sendo um termo contemporâneo usado para se referir a um conjunto de tecnologias de informação (plataformas, aplicações e processos), que visa facilitar a tomada de decisões em todos os níveis". Complementarmente, os autores supracitados elencam algumas características pertencentes, e complementares ao conceito de BI, a saber:

- Sistema de armazenamento dos dados, DataWarehouse (armazém geral de dados) e os Data Marts (banco de dados de sistemas específicos);

- Sistemas de suporte a decisão ou Sistema de Apoio a Decisão (SAD);

- Sistemas de mineração de dados (data minning) e de análise Multidimensional (OLAP);

- Sistemas de gestão Empresarial (ERP) e de relacionamentos com clientes (CRM).

Segundo Angeloni et al. (2006), a BI pode fornecer uma visão sistêmica do negócio, distribuindo os dados de forma concreta, além de transformar esses dados em informações úteis para a tomada de decisão, assim, a $B I$ permite cruzar dados, visualizar informações de formas diferentes e analisar o desempenho empresarial. A Figura 4 representa a estrutura básica conceitual do uso de dados que levam a decisões mais assertivas.

Figura 4 - O Uso de Dados leva a Decisões mais assertivas

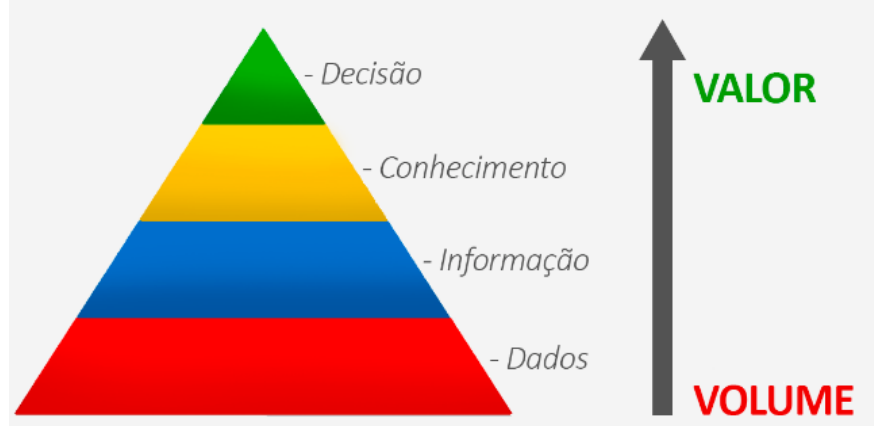

Fonte: Cetax (2019) 
Como se percebe pelo que foi referido, a presença de sistemas de BI é de suma importância na realidade de vida das organizações, tendo em vista que de nada adianta armazenar grande quantidade de dados repetitivos, incompletos e espalhados em vários sistemas dentro das corporações. É necessário apurar os que possuem maior impacto, como forma de destacar os mais valiosos, fazendo com que os dados trabalhem de forma eficiente e garantindo vantagem competitiva.

Para atender a esse mercado de inteligência de informações, as empresas de tecnologia devem oferecer sistemas de BI, com várias funcionalidades e com usabilidade, além de oferecer segurança da informação, sobretudo após a Lei Geral de Proteção dos Dados. Empresas como a Alibaba Cloud, Birst, Board International, Domo e a IBM, são algumas das empresas que ofertam o serviço de BI atualmente. (RICHARDSON et al., 2020)

Na opinião de Primak (2008), a Business Intelligence se caracteriza por promover o trabalho conjunto de componentes que armazenam, analisam e exploram grandes quantidades de dados, de fontes variadas, baseados em hipóteses à procura de padrões e relacionamentos, para obter informações relevantes para o meio empresarial. Turban et al. (2000) endossam essa ideia e completam dizendo que a B.I. também pode ser entendida como um termo que inclui arquiteturas de dados, ferramentas, bancos de dados, aplicações e metodologias diversas.

Por meio da estruturação desses dados, pode-se observar padrões e comportamentos, de forma explícita, de maneira que o analista e/ou o decisor preveja alguns cenários e se antecipe aos fatos. Dessa forma, seria possível minimizar o tempo de resposta e aumentar assertividade da tomada de decisão. Para Franklin, Kenski e Popadivk (2011), o fundamento elementar da assertividade de uma decisão, passa pela validação primária da base de dados. Pois, será por meio dessa base de dados que as saídas gráficas e relatórios serão extraídos e, consequentemente analisados de forma consciente e racional. Com essa estrutura estabelecida, a possibilidade de erros será controlada.

\section{METODOLOGIA}

Esta pesquisa trata de um estudo descritivo, quantitativo e exploratório. Incialmente foi realizado um breve Referencial Teórico sobre COVID-19, Business Intelligence e Power BI. Em seguida, foram coletados dados secundários do Governo do Estado da Paraíba, através da plataforma https://superset.plataformatarget.com.br/superset/Dashboard/vacinas/, sobre a distribuição da $1^{\text {a }}$ dose de vacina contra o COVID-19 em todo o Cariri Paraibano, até o dia 20/05/2021, considerando a Fase 1 da vacinação contra o COVID-19.

Os dados coletados na plataforma supracitada foram: População segundo IBGE, População Alvo, Cidade; Grupo de atendimento, Quantidade de distribuições da $1^{\mathrm{a}}$ dose, Quantidade de distribuições da $2^{\mathrm{a}}$ dose, \%Imunização Global e \%Meta de Imunização. Os dados coletados foram inicialmente tabulados no Microsoft 
Excel, onde foram geradas tabelas. A posteriori, para a criação do Dashboard com os indicadores principais de performance, para o caso em tela, foi utilizado o software Power BI. A Figura 5 ilustra o procedimento metodológico adotado nesta pesquisa.

Figura 5 - Procedimento metodológico para realização da pesquisa

\begin{tabular}{l} 
Referencial Teórico \\
•COVID-19; \\
• Business Intelligence \\
• Power BI. \\
- Coleta de Dados \\
https://superset.plataformatarget.com.br/superset/dashboard/vacinas/ \\
\hline Tabulação dos dados \\
\hline Microsoft Excel > Tabelas \\
\hline Desenvolvimento do Dashboard \\
\hline
\end{tabular}

Fonte: Autores (2021)

\section{RESULTADOS E DISCUSSÃO}

O Cariri Paraibano é dividido é 4 microrregiões: Cariri Ocidental, Oriental, Curimataú Ocidental e Campina Grande. Para além disso, 30 municípios compõem esta região, com características predominantes de clima quente e seco. A Figura 6 ilustra o mapa da Paraíba com suas sub-regiões. 

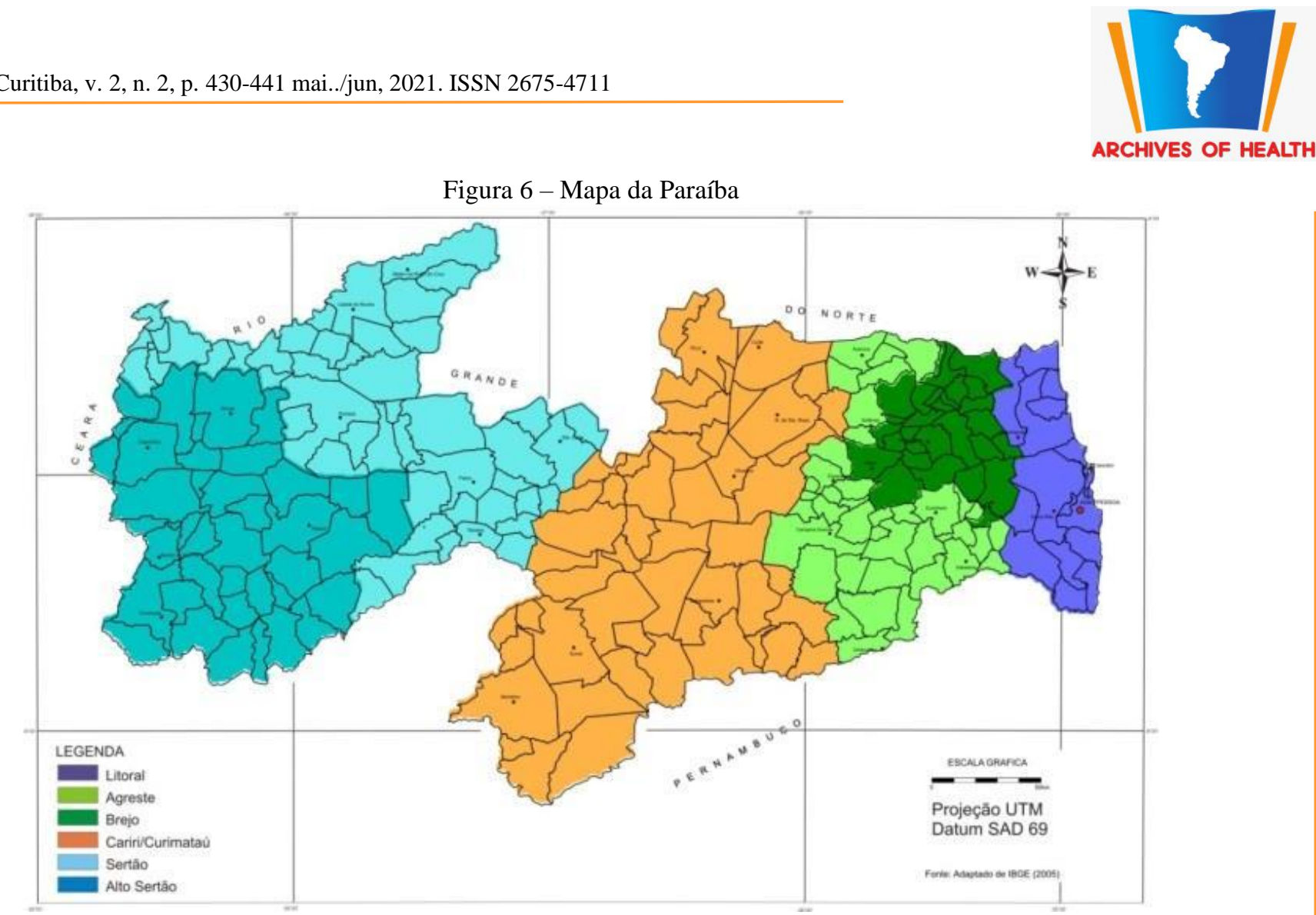

Fonte: Adaptado de https://www.researchgate.net/figure/Figura-1-Regioes-pluviometricamente-homogeneas-do-Estado-daParaiba_fig1_303966738

A Figura 7 mostra a visão geral do Dashboard desenvolvido no Power BI. Nela é possível verificar que o painel de controle oferece, em seu lado esquerdo, dois filtros de pesquisa: cidade e microrregião. Complementarmente, ao selecionar um destes filtros, o mapa da região escolhida será mostrado na tela imediatamente abaixo dos filtros.

Figura 7 - Visão Geral do Dashboard

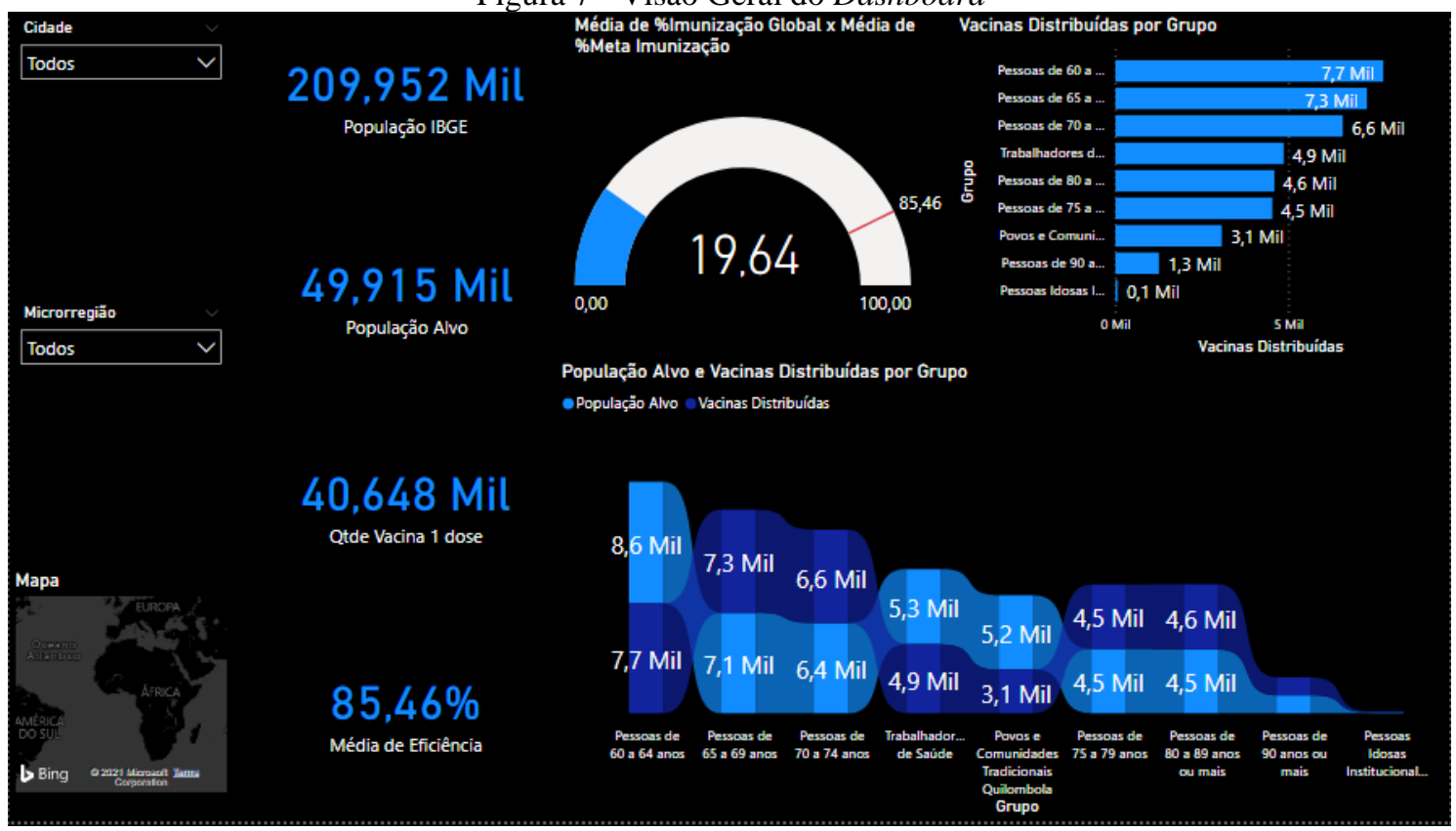

Fonte: Autores (2021) 
Para a tela inicial do Dashboard, conforme mostra a Figura 7, pode-se verificar ainda, através dos cartões, a população total da região do Cariri, segundo o Instituto Brasileiro de Geografia e Estatística (IBGE), a população alvo total para a vacinação, a quantidade de doses distribuídas e a eficiência média de vacinação atingida em todo o Cariri.

Na parte superior central do Dashboard é possível verificar um indicar do percentual médio de Imunização Global x Percentual meta de imunização. A Figura 8 ilustra o caso supracitado.

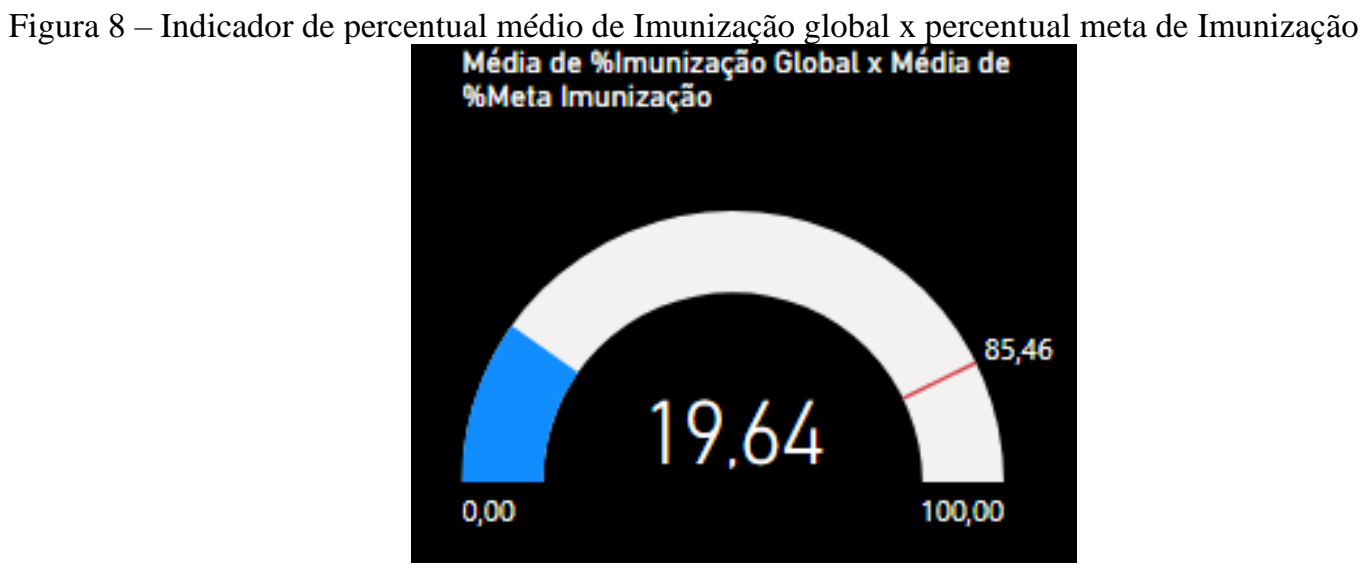

Fonte: Autores (2021)

Conforme mostra a Figura 8, pode-se perceber que a média de imunização global do Cariri contra a COVID-19 está em 19,64\%, ao passo que a média desejada pelos Ministério da Saúde é de 85,46\%.

Por sua vez, a Figura 9 ilustra o Gráfico de Faixas, que mostra o comparativo entre o total de doses distribuídos por grupo e o quantitativo de pessoas por grupo da população alvo. Dele, pode-se inferir, por exemplo, que para o grupo de pessoas de 60 a 64 anos, foram distribuídas 7,7 mil primeiras doses da vacina, ao passo que o a população alvo estimada para este grupo era de 8,6 mil pessoas. Portanto, 900 pessoas do grupo supracitado ficaram sem vacina nesta primeira rodada de distribuição. De maneira análoga, a mesma situação se repete para os grupos de pessoas com 65 a 69 anos, 70 a 74 anos, Trabalhadores de Saúde, Povos e Comunidades Tradicionais Quilombolas. 
Figura 9 - População Alvo x Vacinas distribuídas por grupo

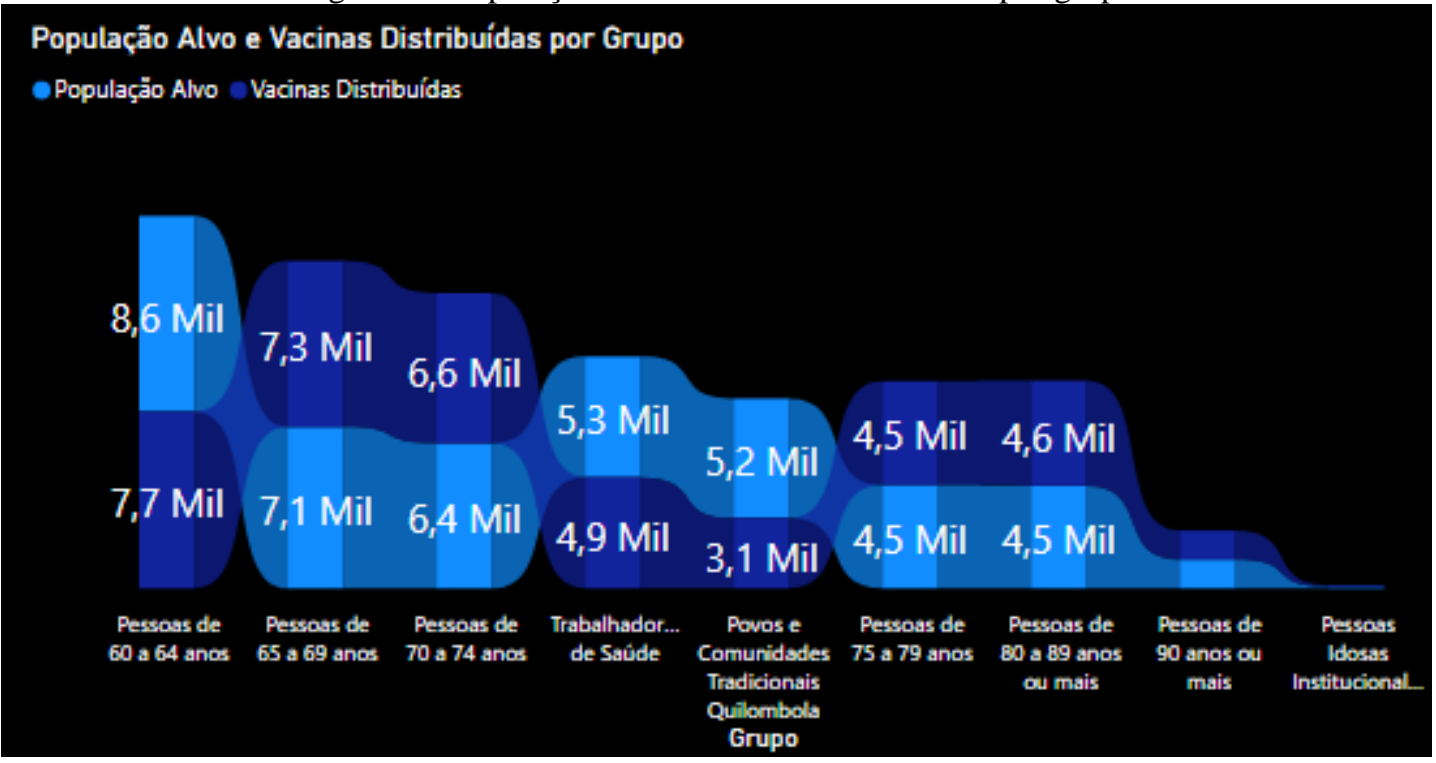

Fonte: Autores (2021)

No grupo de 75 a 79 anos, percebe-se que o número de doses distribuídas de vacina foi exatamente o mesmo que a população alvo. Para o caso das pessoas com 80 a 89 anos ou mais, pode-se visualizar que foram distribuídas mais vacinas do que a população alvo inicial.

A Figura 10 mostra o Gráfico de distribuição de vacinas por tipo de Grupo. Dela pode-se constatar aqueles grupos que foram mais beneficiados com o recebimento da $1^{\text {a }}$ dose de vacina contra o COVID-19, sendo pessoas de 60 a 64 anos as mais beneficiadas e pessoas idosas institucionalizadas as que menos receberam doses de vacinas.

Figura 10 - Vacinas distribuídas por grupo

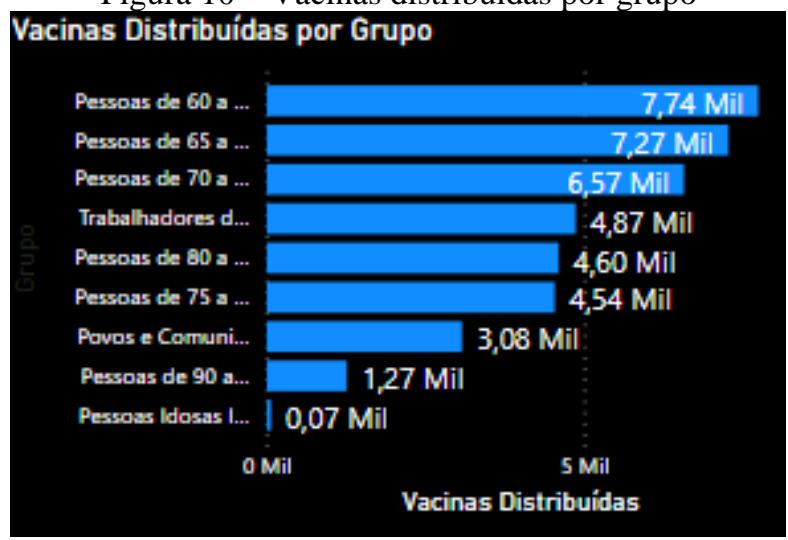

Fonte: Autores (2021)

A Figura 11 mostra o Dashboard com o filtro de cidade aplicado. Neste caso, selecionou-se para o exemplo a maior cidade do Cariri paraibano, Monteiro, com 33433 mil habitantes. Pode-se perceber que a média de eficiência de distribuição da $1^{\text {a }}$ dose de vacina foi de $76,03 \%$ e que 5838 mil doses foram distribuídas. 
Para além disso, o percentual médio de imunização global do munícipio está em 17,46\%, dos 76,03\% esperados, e, pelo Gráfico de faixas, o grupo de 60 a 64 anos não recebeu 200 doses da $1^{\text {a }}$ vacina.

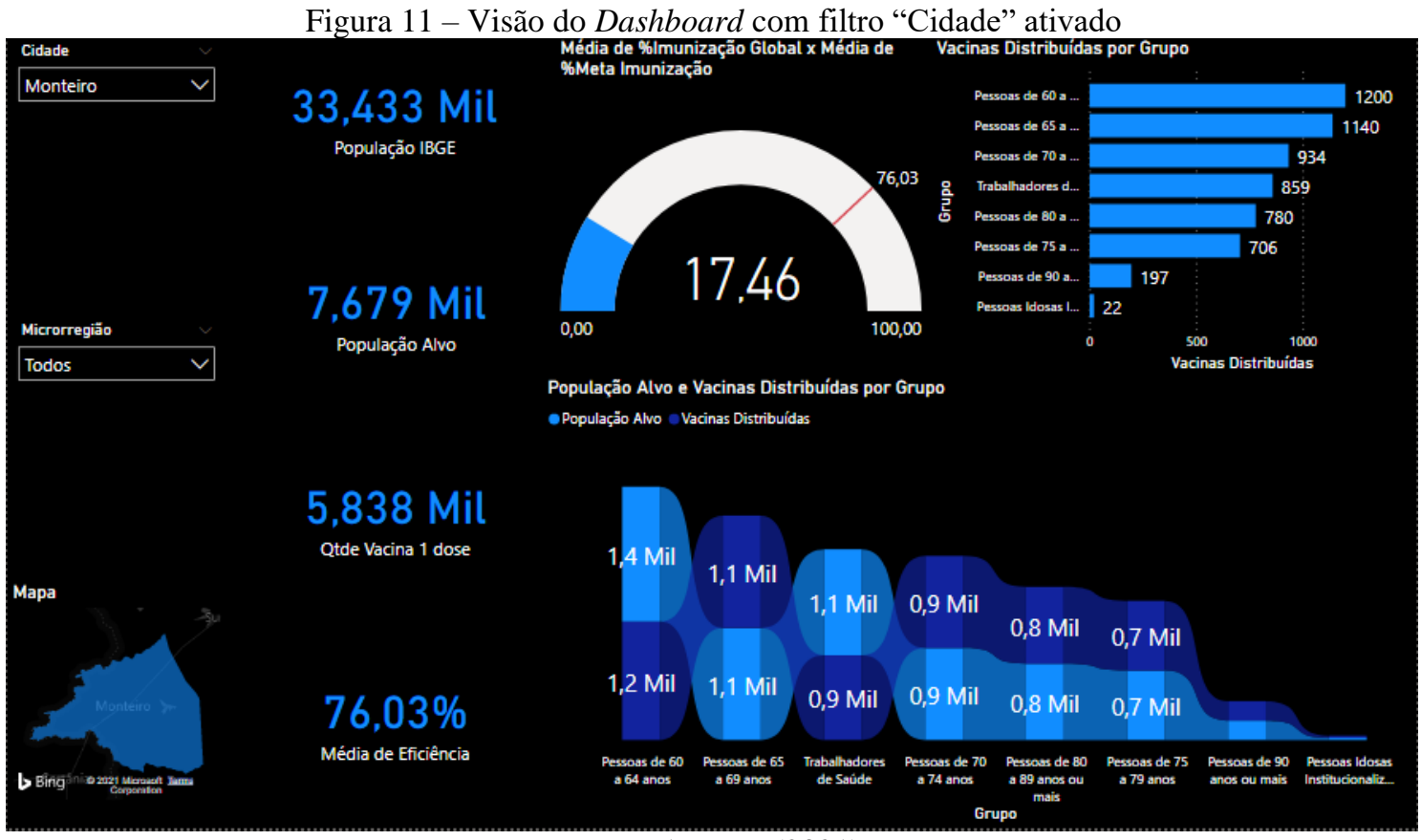

Fonte: Autores (2021)

Ao aplicar os filtros por microrregião, temos os seguintes dados, conforme ilustra a Tabela 1. Da Tabela 1 pode-se constatar que o Cariri Ocidental, composto por 18 municípios, teve a maior quantidade de doses distribuída e possui o maior percentual de imunização global quando comparado com as outras regiões. Em seguida, o Cariri Oriental, composto por 10 municípios. As regiões do Curimataú Ocidental (1 município) e Campina Grande (1 município) foram as com menor quantidade de doses distribuídas (valor absoluto).

Tabela 1 - Sumário por Microrregião

\begin{tabular}{|l|c|c|c|c|c|}
\hline \multicolumn{1}{|c|}{ Microrregião } & $\begin{array}{c}\text { Populaçã } \\
\text { IBGE }\end{array}$ & População Alvo & $\begin{array}{c}\text { Qtde Doses } \\
\text { Distribuídas }\end{array}$ & $\begin{array}{c}\text { \%Imunização } \\
\text { Global }\end{array}$ & $\begin{array}{c}\text { \%Meta } \\
\text { Imunização }\end{array}$ \\
\hline Cariri Ocidental & $\mathbf{1 3 4 3 0 8}$ & $\mathbf{3 4 1 8 9}$ & $\mathbf{2 7 4 7 4}$ & $\mathbf{2 0 , 4 2}$ & $\mathbf{8 4 , 8 8}$ \\
\hline Cariri Oriental & $\mathbf{5 3 4 0 6}$ & $\mathbf{1 1 2 4 6}$ & $\mathbf{9 5 5 0}$ & $\mathbf{1 8 , 8 9}$ & $\mathbf{8 7 , 3 4}$ \\
\hline Curimataú Ocidental & $\mathbf{1 5 1 0 2}$ & $\mathbf{3 0 5 4}$ & $\mathbf{2 4 5 1}$ & $\mathbf{1 6 , 2 3}$ & $\mathbf{8 0 , 2 6}$ \\
\hline Campina Grande & $\mathbf{7 1 3 6}$ & $\mathbf{1 4 2 6}$ & $\mathbf{1 1 7 3}$ & $\mathbf{1 6 , 4 4}$ & $\mathbf{8 2 , 2 6}$ \\
\hline
\end{tabular}

Fonte: Autores (2021)

A Figura 11 mostra o Dashboard com o filtro "Microrregião" ativado para o Cariri Ocidental. 
Figura 11 - Visão do Dashboard com filtro "Microrregião" ativado

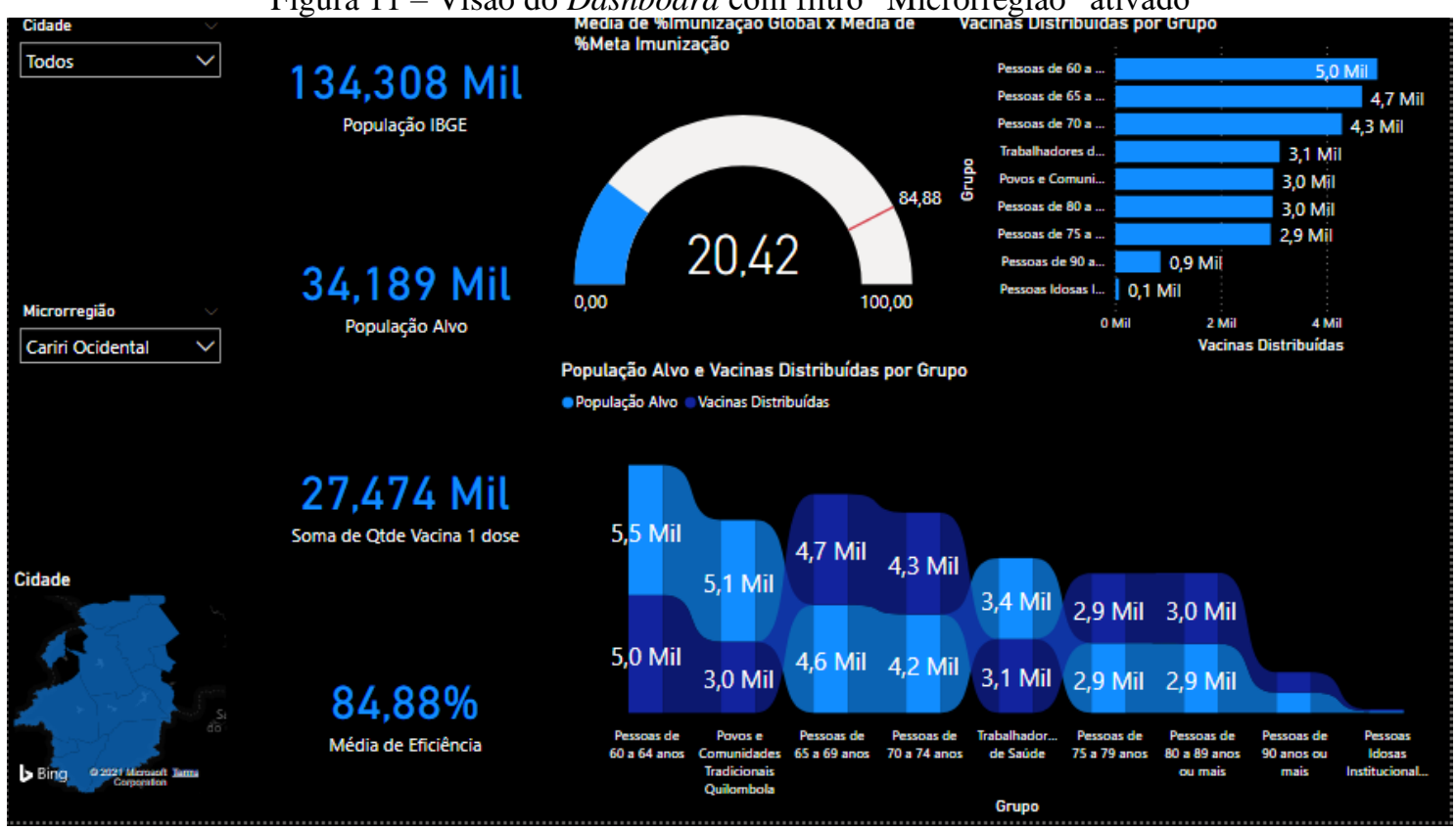

Fonte: Autores (2021)

\section{CONSIDERAÇÕES FINAIS}

Esse trabalho teve como objetivo analisar, a partir da construção de um Dashboard em Power BI, a distribuição de vacinas na Fase 1 no Cariri Paraibano. Através dos indicadores de desempenho disponibilizados no Dashboard desenvolvido, foi possível acompanhar as variações de distribuições de vacinas, público alvo atingido, grupos atingidos e percentuais de eficiência com relação a taxa de vacinação da $1^{\mathrm{a}}$ dose e imunização global x imunização esperada. Diante dos resultados obtidos no desenvolvimento deste trabalho, avalia-se positivamente a arquitetura de solução para administração das informações acerca do objeto de estudo, demonstrando que o uso de Business Intelligence, associado a criação de Dashboard, pode possibilitar aos gestores públicos do Cariri decisões mais acertadas sobre como, onde e quando distribuírem as vacinas, gerando, portanto economia de tempo e esforço no processo de tomada de decisão. 


\section{REFERÊNCIAS}

ANGELONI, M. T. \& Reis, E. S. (2006) Business Intelligence como Tecnologia de Suporte a Definição de estratégias para melhoria da qualidade do ensino. Disponível em: http://www.anpad.org.br/diversos/down_zips/10/enanpad2006-adid-0815.pdf [Acesso em 2020/07/02]

CETAX. Business Intelligence - BI: Tudo o que você precisa saber!. Disponível em: https://www.cetax.com.br/business-intelligence-tudo-o-que-voce-precisa-saber/. Acesso em: 30 abr. 2021

CORDEIRO, Douglas Farias et al. Análise da interação social sobre covid-19 no perfil do ministério da saúde no instagram. Revista Panorama - Revista De Comunicação Social, Goiânia, v. 10, n. 1, p. 19-24, set. 2020. ISSN 2237-1087. Disponível em: <http://revistas.pucgoias.edu.br/index.php/panorama/article/view/8355>. Acesso em: 24 maio 2021. doi:http://dx.doi.org/10.18224/pan.v10i1.8355.

GADELHA, C.A.G. O complexo industrial da saúde e a necessidade de um enfoque dinâmico na economia da saúde. Ciênc Saúde Colet 2003; 8:521-35.

TEMPORÃO, J.G; GADELHA, C.A.G. The Health Economic-Industrial Complex (HEIC) and a new public health perspective. Oxford Research Encyclopedias Glob Public Health. https://oxfordre.com/publichealth/view/10.1093/acrefore/9780190632366.001.0001/acrefore-

9780190632366-e-27. Acesso em $\quad 25 \quad$ maio 2021
» https://oxfordre.com/publichealth/view/10.1093/acrefore/9780190632366.001.0001/acrefore9780190632366-e-27

TODESCO, J. L.; CARRETERO, L. E.; DURAN, A. Business Intelligence. [Slides]. In: CURSO DE BUSINESS INTELLIGENCE. Escuela Complutense Latinoamericana, Florianópolis, 2007.

FRANKLIN, Marcos A.; KENSKI, Victor W.; POPADIUK, Silvio. (2011). Modelo Racional de Tomada de Decisões e seus Pressupostos. Revista de Administração da UNIMEP. v.9, n.3, Setembro/Dezembro2011 ISSN: 1679-5350. Disponível em: http://www.raunimep.com.br/ojs/index.php/regen/article/view/294

LAUDON, Jane P.; Laudon, Kenneth C. Sistemas de informações gerenciais. 11 a . Ed. São Paulo- SP: Pearson Education do Brasil Ltda., 2014. 506 p. ISBN 978-85-4301-507-1.

PRIMAK, F. (2008). Decisões com B.I. (Business Intelligence), 1ªEd. Rio de Janeiro: Ciência Moderna.

RICHARDSON, James; et. al. Magic quadrant for analytics and business intelligence platforms. 2020

TURBAN, E. \& VOLONINO, L. (2013). Business Intelligence e Suporte à Decisão. In A. Evers, (Eds) Tecnologia da Informação para Gestão: em busca do melhor desempenho estratégico e operacional, $8^{\mathrm{a}}$ ed. Porto Alegre: Bookman. p. 468 